\title{
Giant Hypertrophic Gastritis
}

National Cancer Institute

\section{Source}

National Cancer Institute. Giant Hypertrophic Gastritis. NCI Thesaurus. Code C67277.

Gastritis characterized by hypertrophy of the gastric mucosa with creation of giant gastric folds. It is associated with hypoalbuminemia due to albumin loss from the stomach. Signs and symptoms include nausea, vomiting, abdominal pain, edema, and weight loss. 